\title{
Fractional Brownian Motion Approximation Based on Fractional Integration of a White Noise
}

\author{
A. V. Chechkin and V. Yu. Gonchar \\ Institute for Theoretical Physics \\ National Science Center "Kharkov Institute of Physics and Technology" \\ Akademicheskaya St.1, Kharkov 310108, Ukraine \\ and Institute for Single Crystals \\ National Academy of Sciences of Ukraine \\ Lenin ave. 60, Kharkov 310001, Ukraine
}

(March 31, 2021)

\begin{abstract}
We study simple approximations to fractional Gaussian noise and fractional Brownian motion. The approximations are based on spectral properties of the noise. They allow one to consider the noise as the result of fractional integration/differentiation of a white Gaussian noise. We study correlation properties of the approximation to fractional Gaussian noise and point to the peculiarities of persistent and anti-persistent behaviors. We also investigate self-similar properties of the approximation to fractional Brownian motion, namely, " $\tau^{H}$ laws" for the structure function and the range. We conclude that the models proposed serve as a convenient tool for the natural processes modelling and testing and improvement of the methods aimed at analysis and interpretation of experimental data.
\end{abstract}

PACS number(s): 02.50.-r, 05.40.+j

Typeset using REVTEX 


\section{INTRODUCTION.}

Fractional Brownian motion (fBm) is a random process with stationary self-similar increments, which are normally distributed and have an infinite span of interdependence. The general theory of random processes with stationary self-similar increments was developed by Kolmogorov [1] as far back as 1940. Fractional Brownian motions were introduced by Mandelbrot and van Ness as a (relatively) simple family of random functions "that could in some way be expected to be "typical" of what happens in the absence of asymptotic independence“ [2]. In this paper the authors also have introduced fractional Gaussian noise, that is, the stationary process, which is the derivative of the "smoothed" fBm. Three classes of examples have moved these authors to study fractional motions, namely, (i) various economic time series, (ii) $1 / f$, or flicker, noises in electronics and solid state physics, and (iii) the tasks of hydrology, see references in [2]. Over past 30 years the usefulness of fBm's was confirmed repeatedly, and the number of phenomena, which can be viewed as examples of fBm's grows. There are many papers on this subject; here we only mention the monograph [3], which contains a lot of natural examples, the review [4], which deals with the problems of biology and physiology, and the recent monograph [5], in which statistical properties of economical and financial time series are discussed. We also that the description of fractional noises in terms of probability densities requires the use of fractional calculus, which has an ever growing interest among physicists and economists [6].

The ubiquity of fBm's has posed the task of developing approximations, which allow one to simulate fBm's and study their properties in order to develop effective methods for experimental data processing and for the purposes of numerical modelling. Soon after the first paper on fBm [2] Mandelbrot and Wallis have carried out simulation experiments concerning fractional Gaussian noise [7]. They have proposed two approximations to the noise called "Types 1 and 2 approximations". Later on, Voss have proposed an empirical algorithm [8] suited for construction of not only one-dimensional fractional Brownian functions but also fractional Brownian surfaces and volumes. However, we believe, that the ways of 
constructing fractional noises and motions are not exhausted, and various simulation models are needed, each of them may appear to be useful when studying some particular problem.

In this paper we propose a simple model of fBm based on exploitation of spectral properties of fractional Gaussian noise. At first we remind some features, which are essential for our simulation and studies.

\section{SOME PROPERTIES OF FRACTIONAL GAUSSIAN NOISE AND FRACTIONAL BROWNIAN MOTION.}

Below we use the same notations as in original papers by Mandelbrot and van Ness [2] and by Mandelbrot and Wallis [7]. An ordinary Brownian motion with zero mean and unit variance is denoted by $B(t)$, and $\mathrm{fBm}$ by $B_{H}(t)$. The latter is constructed as a moving average of $d B(t)$, in which past increments of $B(t)$ are weighted by the kernel $\left(t-t^{\prime}\right)^{H-1 / 2}$, where $H$ is a parameter satisfying $0<H<1$. For the ordinary Brownian motion $H=1 / 2$. For $H$ lying between 0.5 and 1 the process is called persistent, whereas for $H$ lying between 0 and 0.5 it is called anti-persistent. The introduction of fBm is motivated by the self-similar

properties of its increments, which lead to the " $\tau^{H}$ laws" for the structure function and for the range, that is,

$$
S_{H}^{1 / 2}(\tau)=\left\langle\left(B_{H}(t+\tau)-B_{H}(t)\right)^{2}\right\rangle^{1 / 2}=\tau^{H} V_{H}^{1 / 2}
$$

(an explicit form of $V_{H}$ is unessential here), and

$$
\begin{aligned}
R(t, \tau)= & \sup _{t \leq s \leq t+\tau}\left[B_{H}(s)-B_{H}(t)\right]- \\
& -\inf _{t \leq s \leq t+\tau}\left[B_{H}(s)-B_{H}(t)\right] \stackrel{d}{=} \\
& \stackrel{d}{=} R(0, \tau) \stackrel{d}{=} \tau^{H} R(0,1) .
\end{aligned}
$$

The properties of self-similarity of approximation to $\mathrm{fBm}$ will be checked below.

It is known that the sample paths of fBm are nowhere differentiable. Such irregular functions are best characterized by the Holder exponent or by recently introduced local 
fractional derivative [9]. For the purposes of our paper we note that in applications one always deals with the "smoothed" random process, $B_{H}(t, \delta)$, which may be defined as

$$
B_{H}(t, \delta)=\frac{1}{\delta} \int_{t}^{t+\delta} B_{H}(s) d s
$$

where $\delta$ is the smallest time interval, which is physically relevant for the problem of interest. $B_{H}(t, \delta)$ has a derivative,

$$
B_{H}^{\prime}(t, \delta)=\frac{1}{\delta}\left[B_{H}(t+\delta)-B_{H}(t)\right]
$$

which is called fractional Gaussian noise. It is a stationary process with the covariance, which may be easily determined,

$$
\begin{aligned}
C(\tau, \delta) & =\left\langle B_{H}^{\prime}(t+\tau, \delta) B_{H}^{\prime}(t, \delta)\right\rangle \\
& =\frac{V_{H}}{2 \delta^{2 H}}\left\{(\tau+\delta)^{2 H}-2 \tau^{2 H}+(\tau-\delta)^{2 H}\right\}
\end{aligned}
$$

If $\tau \ll \delta$, it follows from Eq.(5) that

$$
C(\tau, \delta) \propto(2 H-1) \tau^{2 H-2}
$$

and thus, the covariance has the same sign as $H-1 / 2$ and has a very different behavior in a persistent case and in anti-persistent one. The analysis of Eqs.(5), (6) shows that for the former case $C(\tau, \delta)$ is positive for all $\tau$, and

$$
\int_{0}^{\infty} C_{H}(s, \delta) d s=\infty
$$

whereas for the latter $C(\tau, \delta)$ changes sign once from positive to negative at a value of $\tau$ proportional to $\delta$, and one has

$$
\int_{0}^{\infty} C_{H}(s, \delta) d s=0 .
$$

The next property, which serves as the basic one at the initial stage of our simulation, is a power-law behavior of the spectrum of fractional Gaussian noise, 


$$
G(f ; \delta)=4 \int_{0}^{\infty} C_{H}(s, \delta) \cos (2 \pi f s) d s \propto f^{1-2 H}
$$

at $f \delta<<1$.

The spectrum (7) is also quite different for persistent and anti-persistent cases. For the former the main part of the noise energy is concentrated in a low -frequency region, whereas for the latter the spectral intensity grows towards high frequencies.

In numerical simulation one deals with a discrete-time fractional noise, when $t$ is a positive integer and the noise is defined by the sequence of increments $B_{H}(t+1)-B_{H}(t)$

. The correlation function is defined by Eq.(5) with $\delta=1$. The Fourier transform of the discrete-time noise is defined between plus and minus the Nyquist critical frequency $f_{c}=0.5$.

The power-law dependence of the spectrum at small $f$ allows one to simulate fractional Gaussian noise by fractional integration/differentiation of a white noise, with a subsequent construction of fBm.

\section{THE MODELS.}

We remind the relation between the Fourier transforms of the function $X(t)$ and of its fractional integral/derivative $X_{\nu}(t)$ of the $\nu$ order:

$$
\stackrel{\wedge}{X}_{\nu}(f)=\frac{\hat{X}_{\nu}(f)}{(-i 2 \pi f)^{\nu}}
$$

where $\nu$ is positive in case of fractional integration and negative in case of fractional differentiation (to be more accurate, we say about left-side Liouville fractional integral/derivative on the infinite axis [10). If $X(t)$ is a white Gaussian noise, then the spectral density of $X_{\nu}(t)$ is, at least, formally,

$$
G_{\nu}(f) \propto \frac{1}{f^{2 \nu}}
$$

which behaves as the spectrum of fractional Gaussian noise at small $f$, see Eq.(7), if we set $\nu=H-1 / 2, \quad-1 / 2<\nu<1 / 2$. Thus, we use the following way for simulation: 
(i) taking white Gaussian noise $X(t), t$ is an integer, we multiply its Fourier transform by $f^{-\nu},-1 / 2<\nu<1 / 2$.

(ii) making an inverse Fourier transform, we get $X_{\nu}(t)$, which is supposed to approximate fractional Gaussian noise with the index $H=+1 / 2$.

(iii) The process $X_{\nu}^{*}(t)$, which is supposed to approximate fBm, is defined by

$$
X_{\nu}^{*}(t)=\sum_{\tau=1}^{t} X_{\nu}(t)
$$

\section{NUMERICAL RESULTS.}

The results of numerical simulation and analysis are shown in Figs.1-5.

At the left of Fig.1 we show typical examples of the samples of the process $X_{\nu}(t)$ for (a) $\nu=0.4$, (b) $\nu=0$ (white Gaussian noise), and (c) $\nu=-0.4$. The stationarity of the samples was checked by the methods of series and inversions [11]. The figures demonstrate clearly the prevalence of low-frequency components for the case (a), and the prevalence of high-frequency components for the case (c). At the right of Fig.1 the solid lines depict normalized theoretical correlation functions, see Eq.(5), with $\delta=1$, for (a) $H=0.9$, (b) $H=0.5$, and (c) $H=0.1$, whereas the normalized correlation functions

$$
C(\tau)=\frac{\left\langle X_{\nu}(t) X_{\nu}(t+\tau)\right\rangle}{\left\langle\left(X_{\nu}(t)\right)^{2}\right\rangle}
$$

are shown by black points for the same values of as at the left of the figure. The results demonstrate good agreement between correlation function of simulated process with the parameter $\nu$ and theoretical correlation function for the fractional noise with the corresponding index $H=\nu+0.5$. We also see clear difference between correlation functions for the persistent and anti-persistent cases. This difference is the manifestation of the property, which at a qualitative level can be formulated as follows: in the persistent random process the available tendency is supported, whereas in the anti-persistent process the opposite tendency prevails [2] [3]. We also note that the correlation functions indicated by black points are estimated 
from the samples shown at the left with the use of time averaging. The complementary averaging over a set of realizations diminishes scattering of the points.

It is known, that the wavelet transform is well-suited for analyzing structure of nonstationary processes [12]. In particular, it allows one to study the behavior of processes at different scales and hence it is sometimes called as a mathematical microscope. The wavelet transform $T(a, t)$ of the process $X_{\nu}^{*}(t)$ is written as

$$
T(a, t)=\frac{1}{\sqrt{a}} \int_{-\infty}^{\infty} W\left(\frac{t-t^{\prime}}{a}\right) X_{\nu}^{*}\left(t^{\prime}\right) d t^{\prime},
$$

where $W(t)$ is called analyzing wavelet. In Fig.2 we demonstrate the wavelet transforms $T(a, t)$ of the samples of $X_{\nu}^{*}(t)$ for (a) $\nu=0.4$, and (b) $\nu=-0.4$. We use the "Mexican hat" as analyzing wavelet,

$$
W(t)=\left(1-t^{2}\right) \exp \left(-t^{2} / 2\right)
$$

The low part of the wavelet corresponds to small $a$ values (that is, to high frequencies), whereas the upper part corresponds to large $a$ values (low frequencies).Below each of the wavelet the sample path is shown, which is subjected to wavelet transform. It is seen that the wavelet for anti-persistent case has a clearly visualized structure in a high-frequency region. On the contrary, the wavelet for persistent case demonstrate almost homogeneous background in a high-frequency region. The lines of local maxima demonstrate hierarchial structure, which reflects self-similarity of fBm.

In this paper we do not intend to use wavelets for extracting quantitative characteristics, so, Fig.2 has an illustrative purpose only. However, we note that the wavelet transform being applied to some known functions allows one to establish their multifractality [13].

We study numerically $\tau$-dependence of the structure function of the generated process $X_{\nu}^{*}(t)$,

$$
S(\tau)=\left\langle\left(X_{\nu}^{*}(t+\tau)-X_{\nu}^{*}(t)\right)^{2}\right\rangle \propto \tau^{2 s}
$$

Since for fBm the " $\tau^{H}$ law" is fulfilled, see Eq.(1), we expect that $s$ is close to $\nu+0.5$. In Fig.3 $s$ depending on $\nu$ is depicted by points for the persistent processes and by crosses for 
the anti-persistent ones. The expected relation is indicated by dotted line. We see that the numerical relation is well fitted by the expected line, however, some discrepancies appear for the strongly persistent and strongly anti-persistent processes as well. At the insets the structure function versus time delay $\tau$ is indicated by black points in log-log scale. The slopes of fitted lines give the values of $s$ for $\nu=-0.4$ (top inset) and $\nu=0.4$ (bottom inset), respectively.

We now turn to the study of the range of $X_{\nu}^{*}(t)$. In the empirical rescaled range analysis , that is, at experimental data processing or in numerical simulation the range of the random process is divided by the standard deviation of its increments after subtraction of a linear trend, see Ref. [14]. This procedure, in particular, smooths the variations of the range on different segments of time series. As the result of the empirical rescaled range analysis of experimental data one gets the Hurst exponent of the process. For fBm the Hurst exponent thus obtained must coincide with the parameter $H$, see Eq.(2). Fig.4 demonstrates the application of the rescaled range analysis to the sample paths of $X_{\nu}^{*}(t)$. The index $\nu$ is 0.4 , thus the value of the Hurst exponent, which we expect to get according our way of simulating $\mathrm{fBm}$ is 0.9. In Fig.4a the fluctuations of the range $R$ (thin curve) and those of the standard deviation $\sigma$ of the increments (thick curve) are shown for the case when the total length of the sample is divided into 64 segments, each of $=16$ lengthwise. Below the variations of the ratio $R / \sigma$ are depicted. It is shown that fluctuations of the ratio are smaller than those of the range. This circumstance justifies the use of the ratio in the empirical analysis. In Fig.4b the rescaled range versus time interval $\tau$ is depicted by black points in log-log scale. The slope of the fitted line gives the Hurst exponent 0.83 .

In Fig. 5 the values of the Hurst exponent $H_{\nu}$ obtained by applying the rescaled range analysis to the sample paths of $X_{\nu}^{*}(t)$ are shown by black points for the persistent processes and by crosses for the anti-persistent ones. The dots indicate the line $H_{\nu}=\nu+0.5$, that is, the relation, which we expect to get according our way of simulating fBm. It is shown that (i) fairly good agreement is for intermediate $\nu$, (ii) deviations between the results for $X_{\nu}^{*}(t)$ and the theory for fBm grow to the ends of the interval of $\nu$, and (iii) these deviations are 
larger for the anti-persistent case than for the persistent one.

\section{DISCUSSION.}

Figures 3 and 5 allow one to conclude that in the persistent case there is a better agreement with the expected lines than in the anti-persistent case (with the length of the sample and discretization step being equal for both cases). In other words, in our model the low frequency errors are less important than the high-frequency ones. This conclusion is analogous to that concerning "Type 2 approximation" constructed by Mandelbrot and Wallis [7]. This is not surprising since the way of its constructing in real space is similar to our way of constructing approximation in Fourier space. Moreover, we compare the results of our numerical simulations with those performed with the "Type 2 approximation" and the Voss algorithm [8] and found unessential differences between the results for the exponent of the structure function, see Fig.3, and for the Hurst index, see Fig.5, for the three methods. We may conclude that our method is suited for performing simulation of fBm as well as the "Type 2 approximation" and the Voss algorithm are. In all three models the persistent case is simulated with higher accuracy than the anti-persistent one. Fortunately, the persistent case is prevalent in nature [3], therefore, the method proposed in our paper can be used in various applications. At last, we believe that our method will be useful for direct constructing approximations to non-Gaussian fractional noises, in particular, fractional Levy noises. This topic will be the subject of forthcoming paper.

\section{ACKNOWLEDGMENTS}

This work was supported in finance by National Academy of Science of Ukraine, the

Project "Chaos-2" and by INTAS, the Project 93-1194. The information support within the Project INTAS LA-96-09 is also acknowledged. 


\section{REFERENCES}

[1] A. N. Kolmogorov, C.R. (Doklady) Acad. Sci. URSS 26, 115 (1940).

[2] B. B. Mandelbrot and van Ness, SIAM Review 10, 422 (1968).

[3] J. Feder, Fractals. Plenum Press, New York, 1968.

[4] B. J. West and W. Deering, Phys. Reports 246, 1 (1994).

[5] B. B. Mandelbrot, Fractals and Scaling in Finance. Springer-Verlag, New York, 1997.

[6] F. Mainardi, P. Paradisi and R. Gorenflo, In: Econophysics: an Emerging Science. Ed. J. Kertesz and I. Kondor, Kluwer, Dordrecht 1998, p.39.

[7] B. B. Mandelbrot and J. R. Wallis, Water Resources Research 5, 228 (1969).

[8] R. F. Voss, In: Fundamental Algorithms in Computer Graphics. Ed. R. A. Earnshaw, Springer-Verlag, Berlin, p.805 (1985).

[9] K. M. Kolwankar and A.D.Gangal, Phys. Rev. Lett. 80, 214 (1998).

[10] S. G. Samko, A. A. Kilbas and O. I. Marichev, Fractional Integrals and Derivatives and Some Applications. Nauka i Tekhnika, Minsk, 1987 (Engl. transl. Harwood Academic).

[11] J. S. Bendat and A. G. Piersol, Random Data. Analysis and Measurement Procedures. John Wiley \& Sons, New York, 1986.

[12] A. Grossmann and J. Morlet, SIAM J. Math. Anal. 15

[13] A. Arneodo, E. Bacry, J.F. Muzy, Physica A 213, 232 (1995).

[14] H. E. Hurst, R. P. Black, and Y.M. Sinaika, Long Term Storage in Reservoirs. An Experimental Study. Constable, London, 1965.

[15] I. Simosen, A. Hansen, Phys. Rev. E58, 2779 (1998). 


\section{FIGURES}

FIG. 1. At the left: typical samples of the simulated noise $X_{n}(t)$ for (a) $\nu=0.4$; (b) $\nu=0$ (white Gaussian noise), and (c) $\nu=-0.4$. At the right: normalized correlation functions of the simulated noises with the same $\nu$ (black points). Solid lines indicate normalized theoretical correlation functions, see Eq.(5) with $\delta=1$, for (a) $H=0.9$, (b) $H=0.5$, and (c) $H=0.1$.

FIG. 2. Wavelet transforms of the simulated process $X_{\nu}^{*}(t)$ for (a) $\nu=0.4$, and (b) $\nu=-0.4$. Below each of the wavelets the sample paths subjected to wavelet transform are shown.

FIG. 3. The exponent $s$ in the structure function of the simulated process, see Eq.(11), versus the index $\nu$. Black points indicate persistent case, whereas the crosses indicate the anti-persistent one. The line $s=\nu+1 / 2$ is depicted by dots. At the insets: the square root of the structure function versus time in log-log scale (black squares) for $\nu=-0.4$ (top inset) and $\nu=0.4$ (bottom inset). The slopes of fitted lines give the values of the exponent $s$, see the main figure.

FIG. 4. (a) Variations of the range of $X_{\nu}^{*}(t)$ (thin curve), of the standard deviation of $X_{\nu}(t)$ (thick curve), and of their ratio (below) at the different time segments. (b) Rescaled range versus time interval in log-log scale (black points). The slope of the fitted line gives the Hurst index 0.83 .

FIG. 5. Plots of the Hurst exponent $H_{\nu}$ versus $\nu$ for the persistent case (black squares) and anti-persistent one (crosses). The line $H_{\nu}=\nu+1 / 2$ is indicated by dots. 

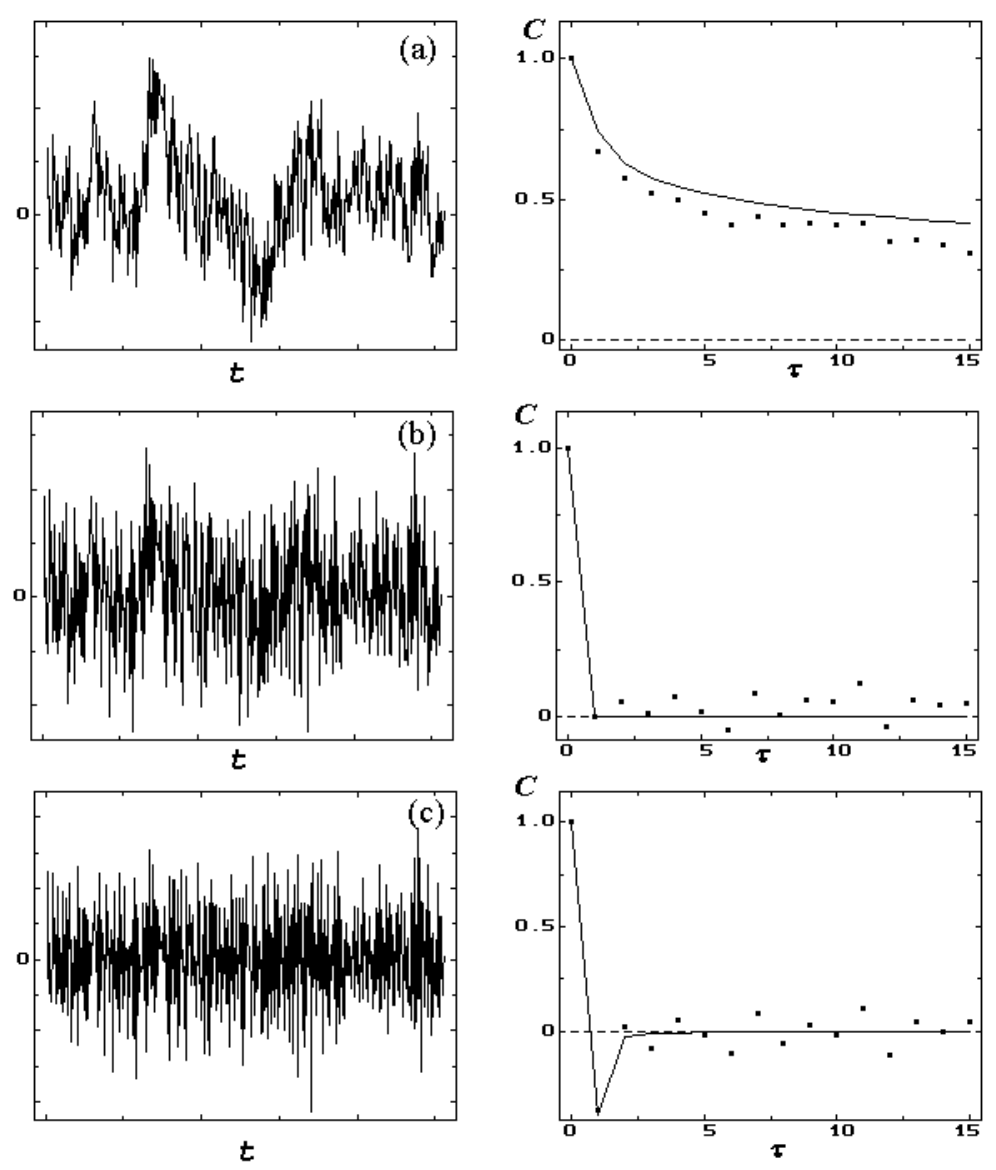

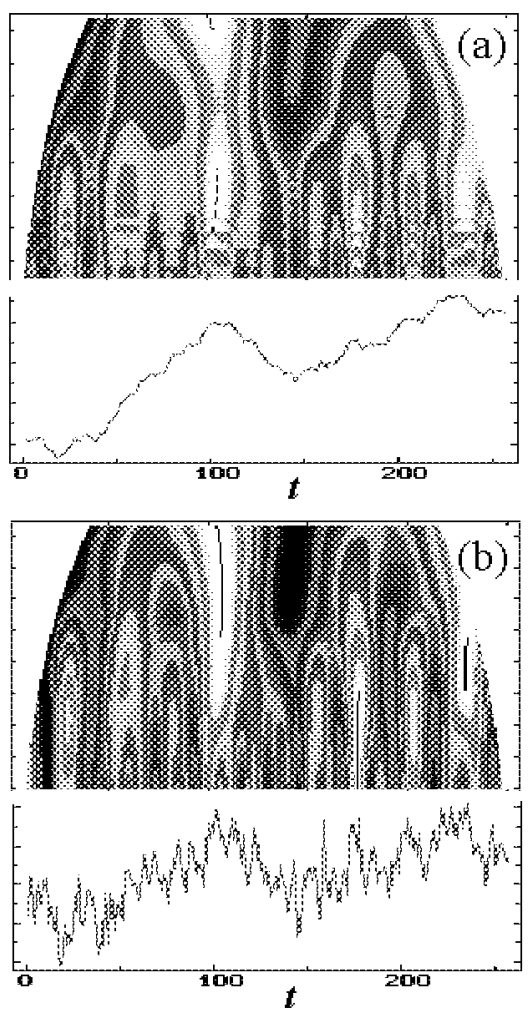


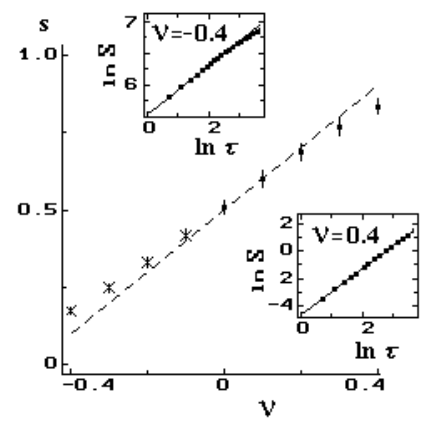




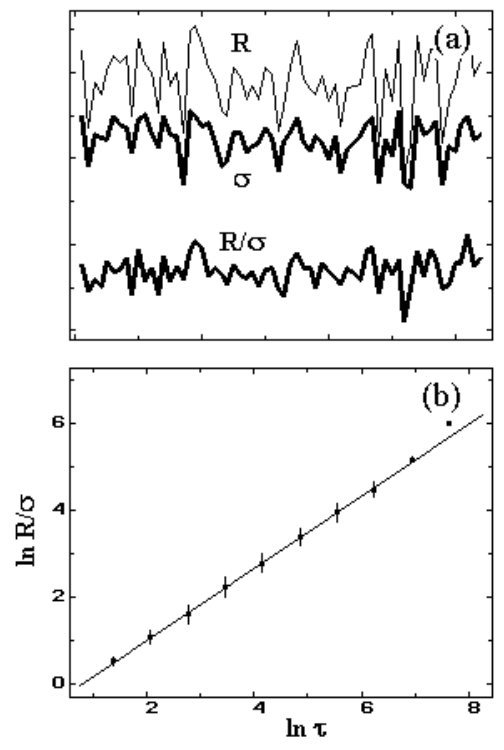




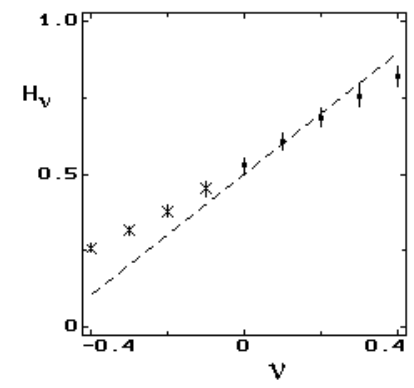

